\title{
A long range, energy efficient Internet of Things based drought monitoring system
}

\author{
Van-Phuc Hoang ${ }^{1}$, Minh-Hong Nguyen ${ }^{2}$, Thanh Quan Do ${ }^{3}$, Dinh-Nhan Le ${ }^{4}$, Du Duong Bui ${ }^{5}$ \\ 1,2,3,4Le Quy Don Technical University, 236 Hoang Quoc Viet Str., Hanoi, Vietnam \\ ${ }^{5}$ National Center for Water Resources Planning and Investigation (NAWAPI), Hanoi, Vietnam
}

\begin{tabular}{|c|c|}
\hline Article Info & ABSTRACT \\
\hline Article history: & \multirow{5}{*}{$\begin{array}{l}\text { The climate change and global warning have been appeared as an emerging } \\
\text { issue in recent decades. In which, the drought problem has been influenced } \\
\text { on economics and life condition in Vietnam. In order to solve this problem, } \\
\text { in this paper, we have designed and deployed a long range and energy } \\
\text { efficient drought monitoring based on IoT (Internet of Things) for real time } \\
\text { applications. After being tested in the real condition, the proposed system has } \\
\text { proved its high dependability and effectiveness. The system is promising to } \\
\text { become a potential candidate to solve the drought problem in Vietnam. }\end{array}$} \\
\hline Received May 30, 2019 & \\
\hline Revised Oct 1, 2019 & \\
\hline Accepted Oct 11, 2019 & \\
\hline Keywords: & \\
\hline
\end{tabular}

Environment Monitoring

Internet of Things

LORA

Smart Water

Copyright $@ 2020$ Institute of Advanced Engineering and Science. All rights reserved.

\section{Corresponding Author:}

Van-Phuc Hoang,

Department of Microprocessor Engineering,

Le Quy Don Technical University,

236 Hoang Quoc Viet Str., Hanoi, Vietnam.

Email: phuchv@lqdtu.edu.vn

\section{INTRODUCTION}

In recent years, Internet of Things (IoT) is the key word that there are a large number of people looking for and it is a part of the fourth industrial revolution. Many researchs [1-5] related to this subject have been performed with many different areas, such as healthcare, transportation, smart infrastructure, smart city, etc. Howerver, most of these applications have different requirements, operate in different environments, so all equipment of each application have to be designed the most optimal to achieve the highest effectiveness. Especially, one of these applications is smart environment monitoring system based on IoT that can provide real time data. In the past few decades, there have been a large number of researchers focus on applications related to Wireless Sensor Networks (WSNs), Wireless Body Area Networks (WBANs), IoT with a variety of different platforms [6-33]. In these designs, microcontrollers (MCU) which are often ultra-low power chips were chosen to reduce the systempower consumption and MSP430 family from Texas Instrument (TI) is a good choice [8-10, 13]. Moreover, some transceivers were used in these works, including Wi-Fi module, bluetooth module, Radio Frequency (RF) module with many different protocols. Especially, authors in [10] presented the comparison of different wireless transmission modules to give the best choice for a detailed application. In addition, in recent years, smart cities have concerning in many countries, so Wi-Fi will cover most of place in the future. Therefore, using Wi-Fi modules as transceiver should be used in applications related to WSN, WBAN, IoT. With each application which has different functions, sensors will be chosen to ensure the lowest power consumption and the highest accuracy. However, to provide long range communications, energy efficient and real time monitoring, in this paper, LORA technology is used. 
In Vietnam, the drought has appeared as a burning issue and seriously influenced on economics, society and the daily life for recent years. In addition, the drought increases significantly the level and widens the affected area, leading to critical consequence if it is not controlled effectively as reported in [34]. As a result, the early drought warning and monitoring is an essential solution to address this matter. In this work, we have researched, designed and implemented a really full worked-out system based on LORA technology drought for monitoring, consisting of both hardware and monitoring software. After a number of experiments, the system has demonstrated the effectiveness and stable working. The rest of this paper is organized as follows. Section 2 describes the system architecture. Section 3 shows data analysis and processing method. Section 4 presents the implementation results and finally, Section 5 concludes of the paper.

\section{SYSTEM DESIGN}

The proposed IoT based drought monitoring system has three main sectors including slave node, master node and monitor software as shown in Figure 1. These sectors are connected throught an IoT platform with LORA, Wi-Fi and Internet systems. The slave nodes will collect temperature and precipitation information and then transmit this information to master node as shown in Figure 2. Since there have many slave nodes, each node will be assigned a code. When the slave node receives an instruction from the master node, it will transmit data. Consequently, data management of the slave node is more efficient and the slave node power consumption is reduced. On the other hand, the master node roles are to monitor and control the slave nodes, to collect data and then to calculate the drought parameters and transmit this information to website. The website will show the recent temperature and precipitation data of all slave nodes, daily and monthly average temperature and precipitation, and the SPI, PE and J index. All these parameters will be analyzed in order to state the drought level of each area and predict the drought trend in the future.

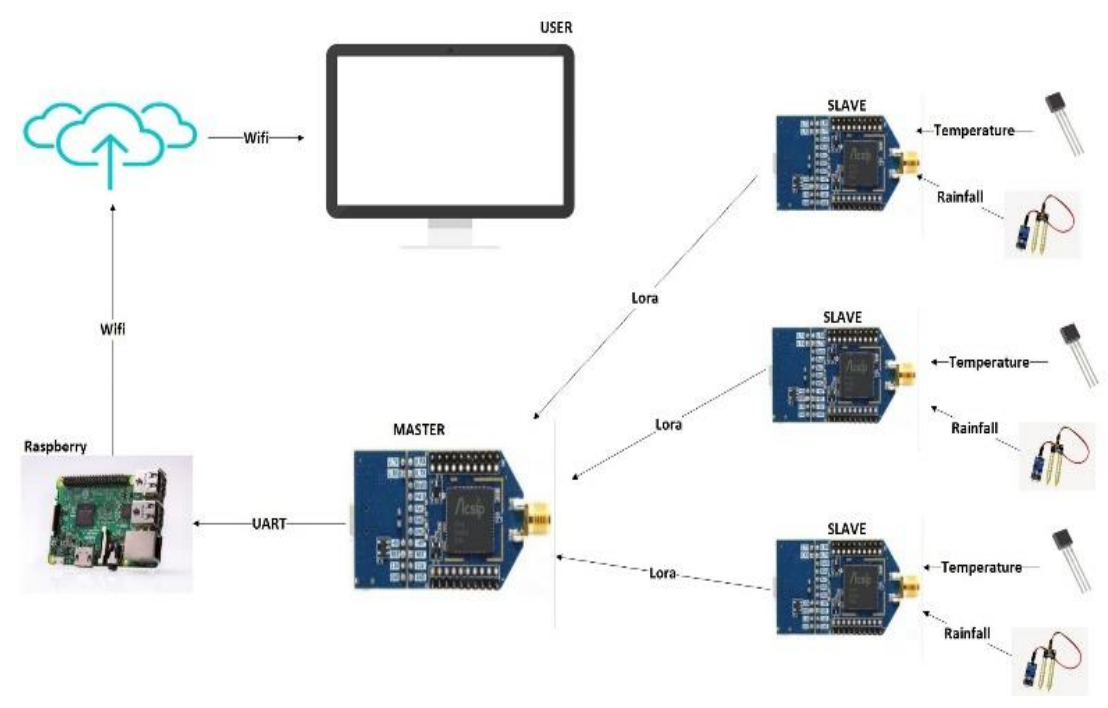

Figure 1. System block diagram of proposed IoT based drought monitoring system

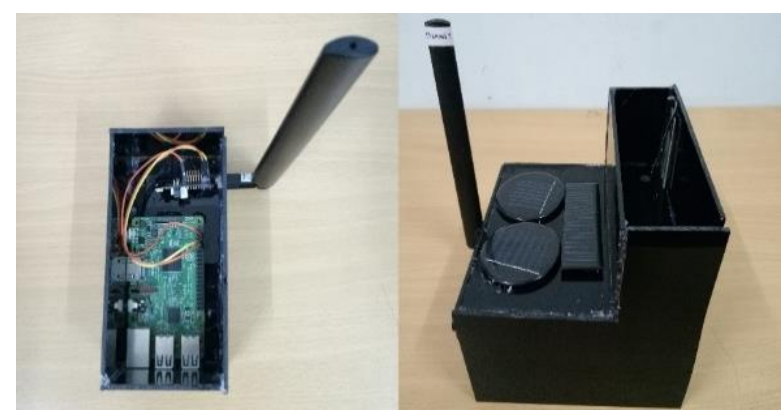

Figure 2. The master (right side) and slave (left side) nodes hardware 
In order to implement the proposed system, the AcSIP LoRa WAN EVK+Antenna KIT S76SXB of the ACSIP Technology Corp was used. This development kit can be considered as a really effective platform for the popose of technical developing and testing of the AcSIP S76S SIP LoRa module employing LoRaWAN protocol as shown in Table 1 [33]. Because USB-to-UART chip is included in the developer board, testing and programming of the MCU becomes more easily. Moreover, since a Micro-USB connector is used in the interface, it can power the modules. In addition, the sensors used in the slave nodes play an important role in the proposed system because they affect directly to accuracy and dependability of the system. In the proposed system, the DS18B20 sensor was used to measure the temperature due to its high accuracy and low power consumption. In order to measure the precipitation, the system employs a box to contain water. This box contains the sensor to measure the water level and has a normal closed valse. The valse will be opened daily to evaluate the daily precipitation.

Table 1. LoraWAN parameters

\begin{tabular}{ll}
\hline LoraWAN parameters & Value \\
\hline Frequency & $433 \mathrm{MHz}$ \\
Bandwidth & $125 \mathrm{KHz}$ \\
Spreading Factor & 10 \\
Coding Rate & $4 / 5$ \\
Tx Power & $7 \mathrm{dBm}$ \\
Sensitivity & $-137 \mathrm{dBm}$ \\
Payload length & $64 \mathrm{bytes}$ \\
CRC on payload & Enable \\
Distance & $16 \mathrm{~km}$ \\
\hline
\end{tabular}

\section{IMPLEMENTATION RESULTS AND ANALYSIS}

\subsection{Evaluating precipitation}

In the proposed system, three main precipitation indexes were analyzed, the Standardized Precipitation Index (SPI) [34], the Precipitaion Effectiveness Index (P-E index) [35] and the De Martonne aridity index (Iar-DM) [36]. These indexes will be clarified briefly as follows. The first index, SPI, can be defined as following equation:

$$
S P I=\frac{P \quad \bar{P}}{}
$$

where $\mathrm{P}$ and $\bar{P}$ are the precipitation and the average precipitation (in $\mathrm{mm}$ ) in defined time period, respectively. The $\sigma$ parameter is the standard deviation of the precipitation. In fact, the SPI can determine the wetness value in different time periods as 1, 3, 6 months; 1, 2 years, and so on based on the application of user, ranging from -2.0 to +2.0 as shown in Table 2 . In the proposed system, the time period was chosen as one month.

Table 2. The range of the SPI value

\begin{tabular}{ll}
\hline SPI value & Climate condition \\
\hline$\geq 2$ & Extremely wet \\
$1.50 \div 1.99$ & Very wet \\
$1.00 \div 1.49$ & Moderately wet \\
$-0.99 \div 0.99$ & Near normal \\
$-1.49 \div-1.00$ & Moderately dry \\
$-1.99 \div-1.50$ & Severely dry \\
$\leq-2$ & Extremely dry \\
\hline
\end{tabular}

The second index, (P-E index), was introduced by Thornthwaite and used to analyze the climate. The index is defined by the following equation:

$$
\mathrm{P}-\mathrm{E}={ }_{i=1}^{12} 115\left(\frac{P}{T 10}\right)^{10 / 9}
$$

Where $\mathrm{P}$ and $\mathrm{T}$ are the mean monthly precipitation in inches and temperature in $\mathrm{F}$ as shown in Table 3. 
Table 3. The range of the P-E index value

\begin{tabular}{cc}
\hline P-E value & Climate condition \\
\hline$\geq 128$ & Wet \\
$64 \div 127$ & Humid \\
$31 \div 64$ & Sub-hummid \\
$16 \div 31$ & Semi-arid \\
$\leq 15$ & Arid \\
\hline
\end{tabular}

The third index, Iar-DM, is the suggestive indicator for the characterization of the aridity index which is introduced by De Martonne, being clarified by the following equation:

$$
\operatorname{Iar}-D M=\frac{12 P}{T_{m}+10}
$$

Here, $\mathrm{P}$ and $T_{m}$ are the total monthly precipitation and the mean monthly temperature, respectively. In the equation (3), the additional value of $10^{\circ} \mathrm{C}$ is added to the mean monthly temperature in order to make positive results in some regions having the negative average annual temperatures such as mountainous and desert areas. The Iar-DM index is basically used to evaluate the climate and subsequently used in the clarification of soil hydrologic regime as shown in Table 4.

\subsection{The proposed method of precipitation evaluation}

In our experimental system, there are one master node and three slave nodes in which each slave node is assigned by one code. In this paper, the working process of the proposed system will be illustrated clearly. First of all, the master node frequently sends the slave node code in the circle in which each node code will be sent in given number of minutes as shown in Figure 3a. Second, when the slave node receives its node code, it will measure the temperature and precipitation, transmitting these information to the master node as shown in Figure 3b. The master node controls all the slave nodes and every day the master node send a reset instruction to all the slave nodes in order to collect data for new day. Finally, when the master node receives data from the slave node, it will transmit this data to sever computer as shown in Figure 3a. The sever computer will analyze and calculate the defined indexes and informs the climate condition. All this information will be shown on the website.

Table 4. The range of the Iar-DM index value

\begin{tabular}{cc}
\hline Iar-DM value & Climate condition \\
\hline$\geq 60$ & Very wet \\
$30 \div 60$ & Wet \\
$20 \div 30$ & Mildly wet \\
$15 \div 20$ & Semi-dry \\
$5 \div 15$ & Dry \\
$0 \div 5$ & Very dry \\
\hline
\end{tabular}

\subsection{Power consumption of the system}

For many smart electronic systems, the power consumption is the key factor which determines the dependability and the effectiveness. In the proposed system, the power consumption of the slave node is the most important because they are set far from the control center. On the other hand, the power consumption of the master node and the sever computer is not necessary because they are set in the control center. As a result, in this paper the energy consumption of the slave node will be calculated mathematically and tested in the real system.

In our system, the period of each slave node is 5 minutes (300 seconds) insitting of 290 seconds in waiting mode and 10 seconds in reading and sending mode. Depending on the datasheet of devices, the total current is about $9 \mathrm{~mA}$ in the waiting mode and is $61.4 \mathrm{~mA}$ in the reading and sending mode in which the current of the LoRa s76s kit, DS18b20 sensor and the precipitation sensor are $49 \mathrm{~mA}, 1 \mathrm{~mA}$ and $14.1 \mathrm{~mA}$, respectively. Consequently, the total average current can be calculated approximately as:

$$
I_{0}=\frac{9 * 290+64.1 * 10}{300}=10.8(m A)
$$


In the experimental system, we used two Ultrafire 18600 batteries combined parallel in which each battery capacity is $4800 \mathrm{mAh}$. As a result, the average life time of the slave node will be:

$$
T_{\text {Life }}=\frac{4800 * 2}{10.8}=888.9(\text { hours })=37(\text { days })
$$

In fact, the real average life time of the slave node is about 35 days. This life time is reasonable, however, it does not meet requirement of the modem smart and automatic systems. To overcome this problem, we proposed two types of energy for the slave node that are solar energy and solar energy combined with standby battery, depending on the sunlight condition. After one-year testing, the solar energy has illustrated its high dependability and efficiency.

\subsection{Demonstration system}

In this work, we have designed a complete system consiting of both hardware and software for drought monitoring. After testing in the real condition in three areas in Hanoi, the proposed system has proved its high effectiveness and dependability. In order to produce convenience to user, the Google map is also intergated into our website, consiquently, the user can easily find out information about temperature, precipitation, drought index and drought degree by one click to the slave node area as shown in Figure 4 . After testing the proposed system in three areas in Hanoi (Cau Giay, Phuong Canh and Academy of Finance), the system has produced the dependable information about the temperature and precipitation all the year as in Figures 5-7 and the drought index and the drought degree as in Figures 8-10. By analyzing this information, climate experts can support the government and authority to make and adjust plan to overcome drought problem. The proposed system can be considered as a potential candidate to solve partly the climate change and global warning in order to bring better life for people.

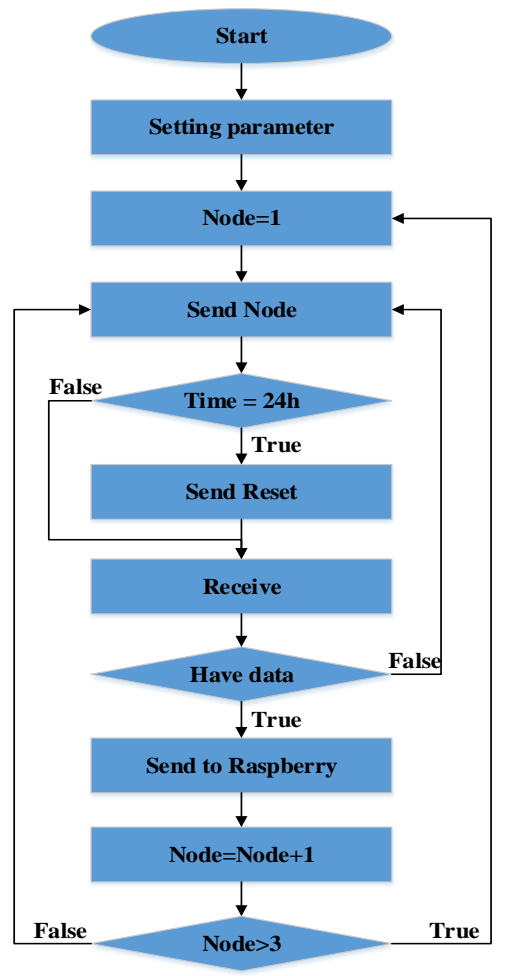

(a)

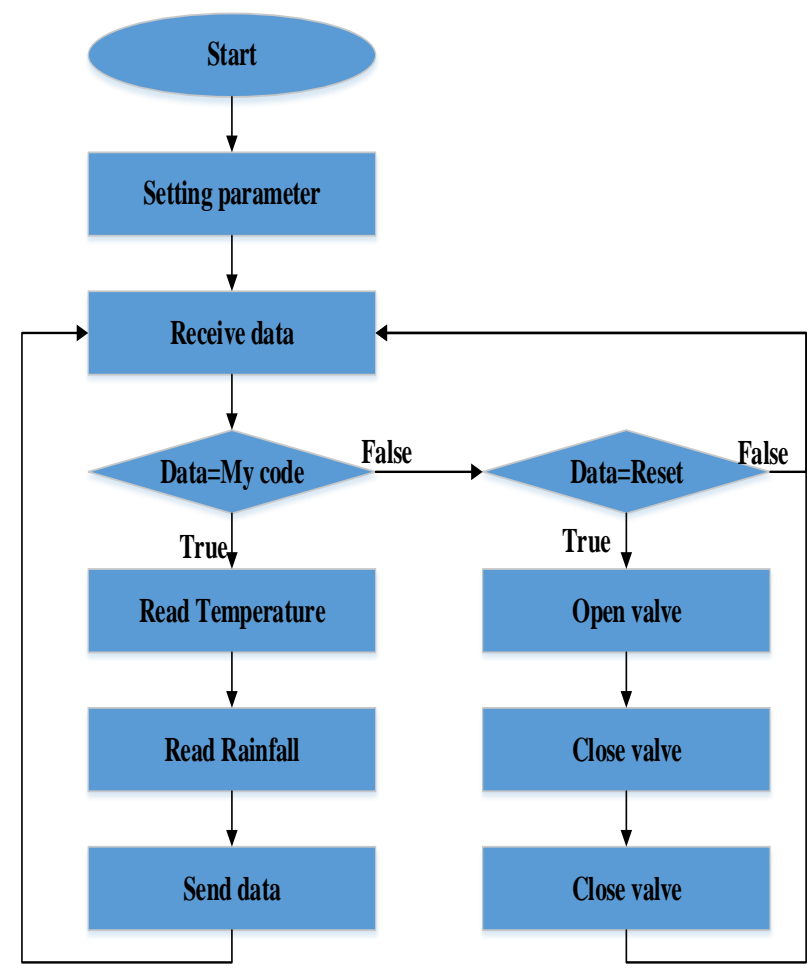

(b)

Figure 3. The working algorithms of the master (a) and slave (b) nodes 


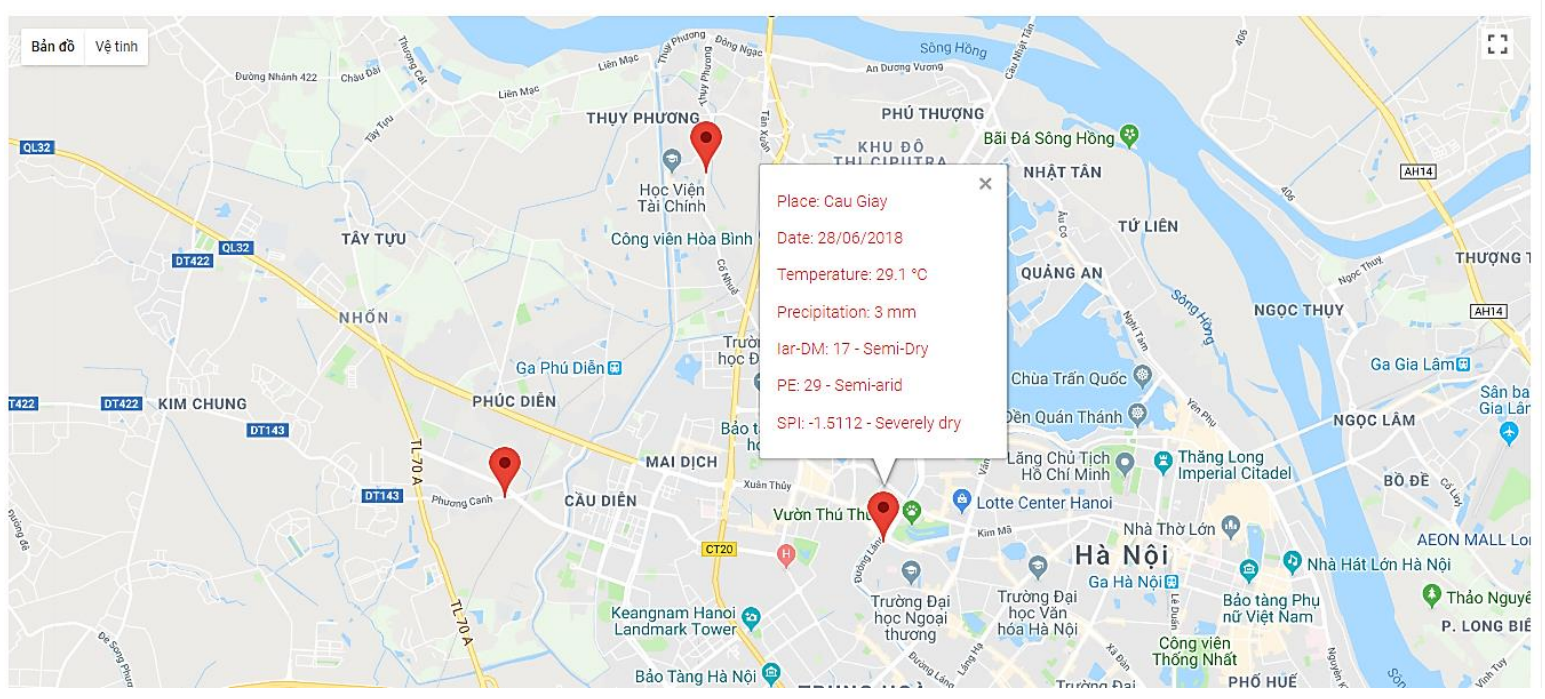

Figure 4. The map of the slave node distribution

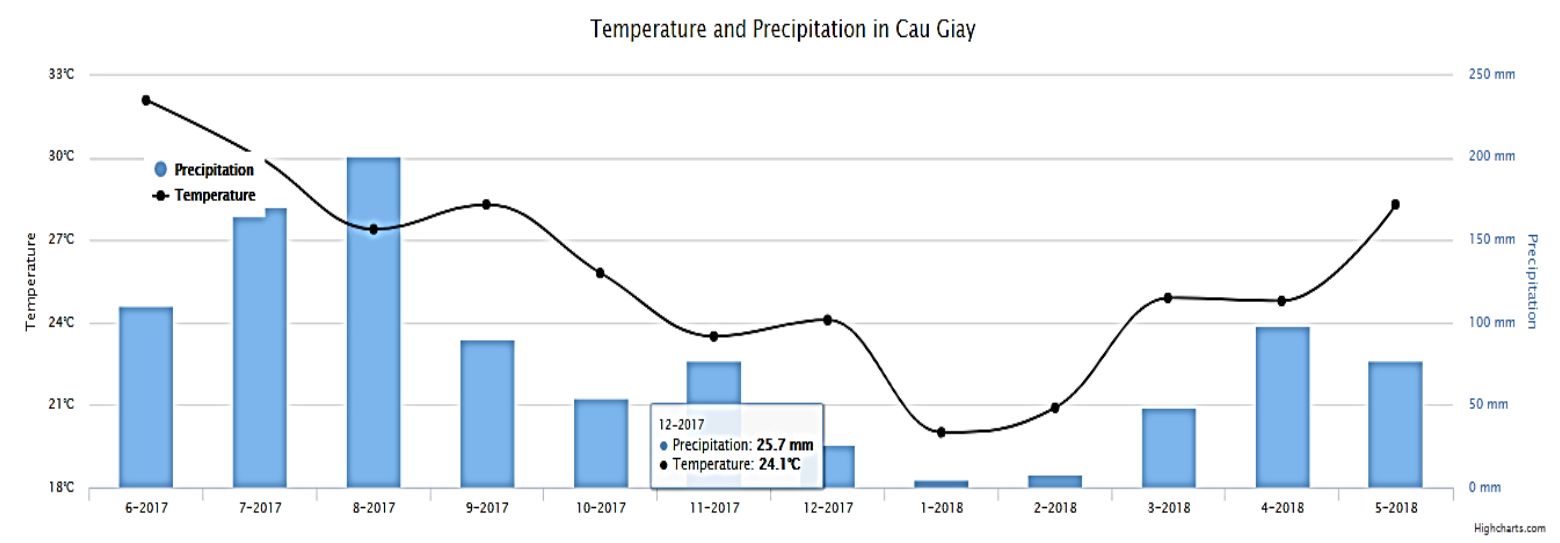

Figure 5. Temperature and precipitaion in Cau Giay district

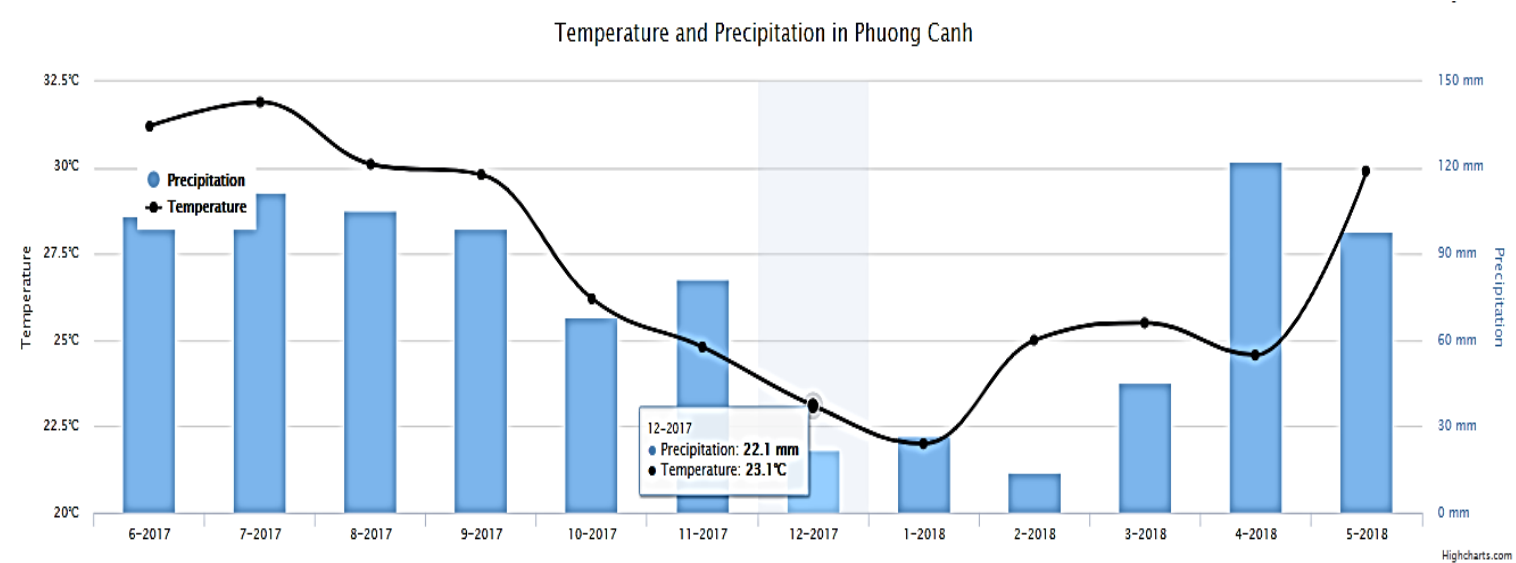

Figure 6. Temperature and precipitaion in Phuong Canh 
Temperature and Precipitation in Academy of Finance

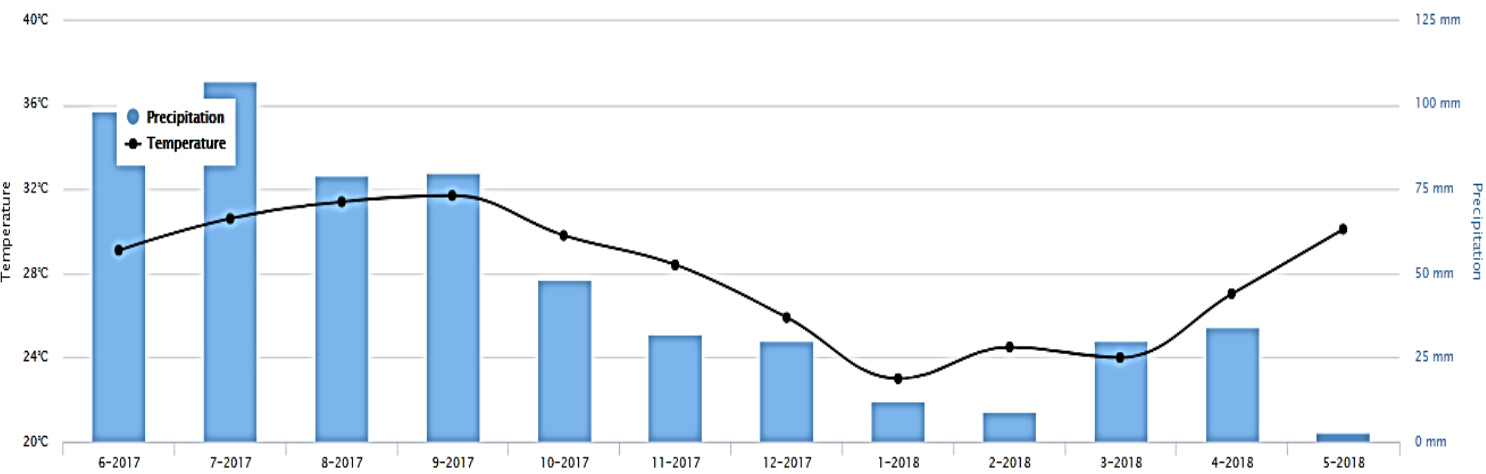

Figure 7. Temperature and precipitaion in Academy of Finance

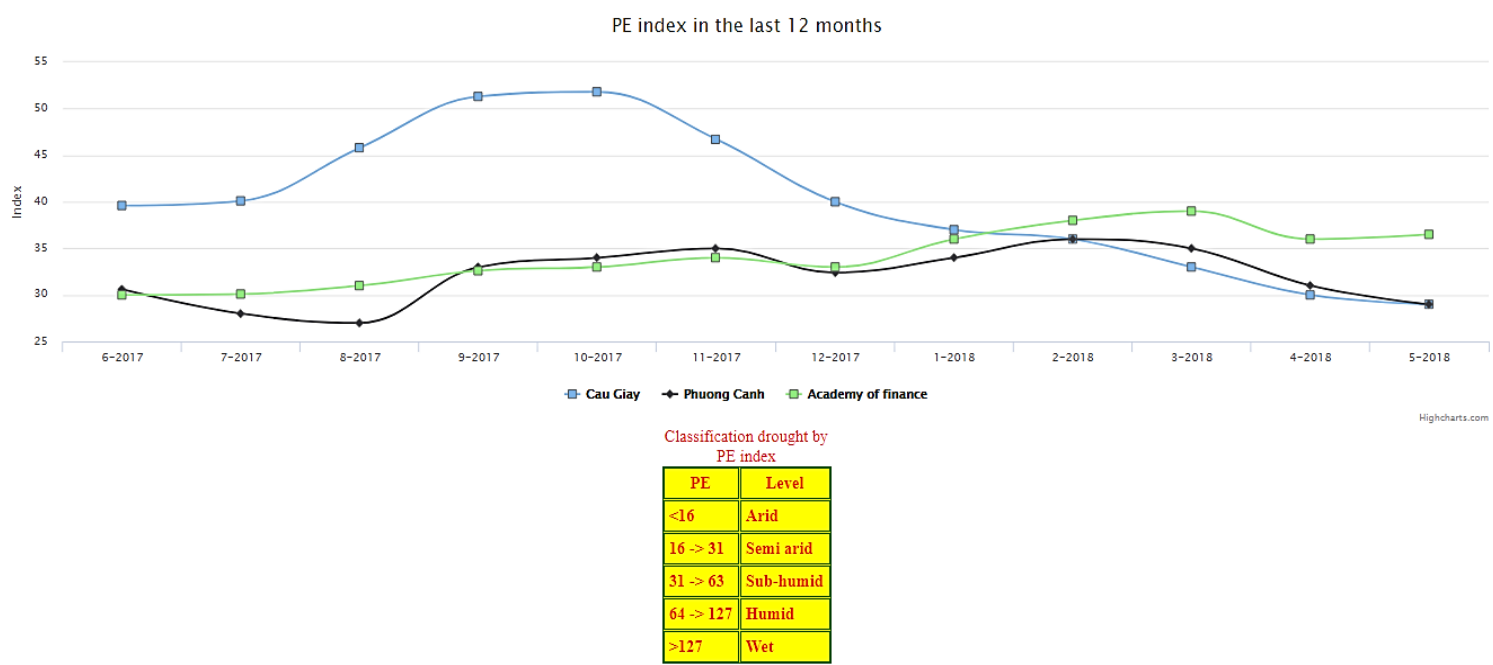

Figure 8. P-E index

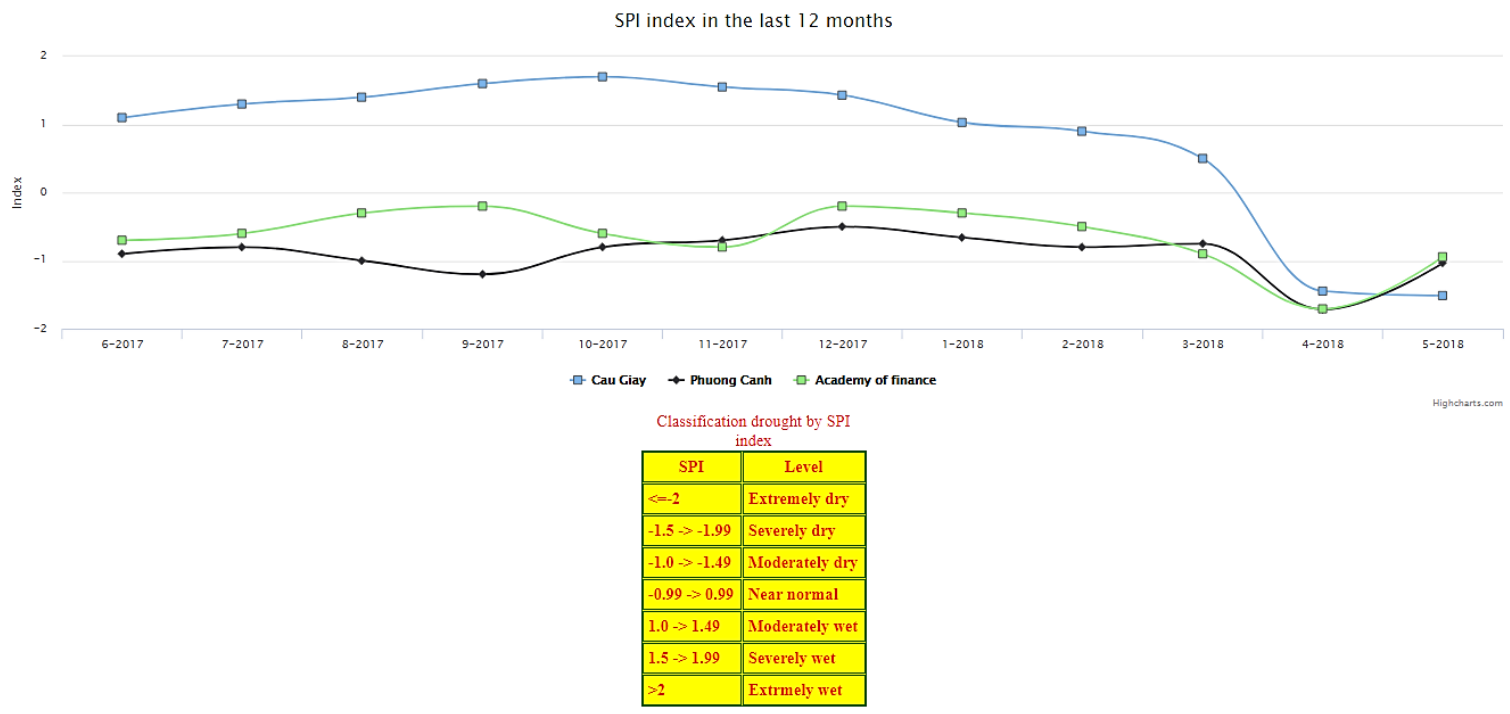

Figure 9. SPI index 


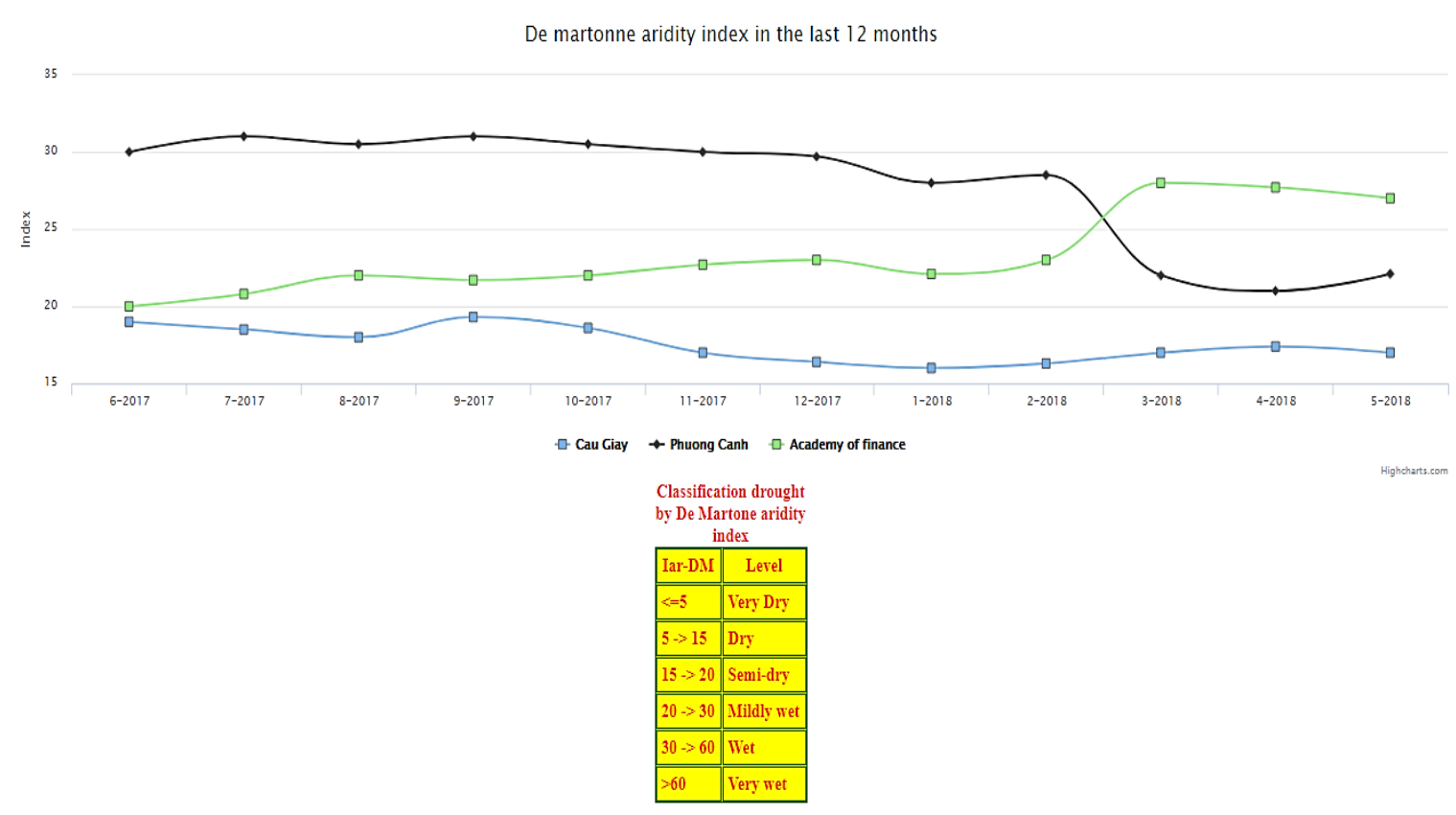

Figure 10. Iar-DM index

\section{CONCLUSION}

In this paper, a complete system based on IoT has been designed and tested for drought monitoring. The proposed system has shown its high dependability and effectiveness. We believe that the proposed system can be a potential solution to support our government to solve the climate change issuses that has been affecting significantly and suffering to economics, society and the life condition in Vietnam. For the future work, the proposed system needs to be tested for more time and area to prove its benefits. We also need to improve protocol to control the slave node more effeciently when the number of the slave node increases to cover a wider area.

\section{ACKNOWLEDGMENT} VINIF.2019.17.

This research is funded by Vingroup Innovation Foundation (VINIF) under the grant number

\section{REFERENCES}

[1] L. Boves and E. den Os, "Speaker recognition in telecom applications," Proceedings. 1998 IEEE 4th Workshop, Interactive Voice Technology for Telecommunications Applications, 1998. IVTTA '98. Torino, pp. 203-208, 1998.

[2] I. V. McLoughlin and Hamid Reza Sharifzadeh, "Speech recognition engine adaptions for smart home dialogues," 2007 6th International Conference on Information, Communications \& Signal Processing, Singapore, pp. 1-5, 2007.

[3] E. Marchetto, F. Avanzini and F. Flego, "An automatic speaker recognition system for intelligence applications," 2009 17th European Signal Processing Conference, Glasgow, pp. 1612-1616, 2009.

[4] K. Selvan, A. Joseph and K. K. Anish Babu, "Speaker recognition system for security applications," 2013 IEEE Recent Advances in Intelligent Computational Systems (RAICS), Trivandrum, pp. 26-30, 2013.

[5] R. Ajgou, S. Sbaa, S. Ghendir, A. Chamsa and A. Taleb-Ahmed, "Robust remote speaker recognition system based on AR-MFCC features and efficient speech activity detection algorithm," 2014 11th International Symposium on Wireless Communications Systems (ISWCS), Barcelona, pp. 722-727, 2014.

[6] O. Hahm, E. Baccelli, H. Petersen and N. Tsiftes, "Operating Systems for Low-End Devices in the Internet of Things: A Survey,” in IEEE Internet of Things Journal, vol. 3, no. 5, pp. 720-734, Oct. 2016.

[7] S. M. R. Islam, D. Kwak, M. H. Kabir, M. Hossain and K. S. Kwak, "The Internet of Things for Health Care: A Comprehensive Survey," in IEEE Access, vol. 3, no. , pp. 678-708, 2015.

[8] A. Kamilaris and A. Pitsillides, "Mobile Phone Computing and the Internet of Things: A Survey," in IEEE Internet of Things Journal, vol. 3, no. 6, pp. 885-898, Dec. 2016.

[9] C. Perera, C. H. Liu, S. Jayawardena and M. Chen, "A Survey on Internet of Things from Industrial Market Perspective," in IEEE Access, vol. 2, pp. 1660-1679, 2014. 
[10] S. H. Shah and I. Yaqoob, "A survey: Internet of Things (IoT) technologies, applications and challenges," 2016 IEEE Smart Energy Grid Engineering (SEGE), Oshawa, ON, pp. 381-385, 2016.

[11] Yan Liu, Lin Huang and Yang Xu, "Embedded system of oil wells monitoring base on GPRS," 2009 Asia-Pacific Conference on Computational Intelligence and Industrial Applications (PACIIA), Wuhan, 2009, pp. 451-454.

[12] S. Duan, Y. Yu, Y. Wan and Q. Wang, “A low-power wireless remote sensor node design based on GPRS,” 2010 International Conference on Computer Application and System Modeling (ICCASM 2010), Taiyuan, 2010, pp. V12-483-V12-488.

[13] YingHui Huang, Xun Huang and Qiang Sun, "The Design of the management system for parking lot based on $2.45 \mathrm{GHz}$ active RFID," 2011 International Conference on Computer Science and Service System (CSSS), Nanjing, 2011, pp. 850-853.

[14] B. Li and J. Zou, "The design of oil drilling wireless data acquisition system," 2011 International Conference on Electrical and Control Engineering, Yichang, 2011, pp. 1810-1813.

[15] Y. Wang, C. Hu, Z. Feng and Y. Ren, "Wireless transmission module comparison," 2014 IEEE International Conference on Information and Automation (ICIA), Hailar, 2014, pp. 902-907.

[16] H. Kim, J. Shin, H. Shin and B. Song, "Design and Implementation of Gateways and Sensor nodes for Monitoring Gas Facilities," 2015 Fourth International Conference on Information Science and Industrial Applications (ISI), Busan, 2015, pp. 3-5.

[17] D. Spirjakin, A. M. Baranov and V. Sleptsov, "Design of smart dust sensor node for combustible gas leakage monitoring," 2015 Federated Conference on Computer Science and Information Systems (FedCSIS), Lodz, 2015, pp. 1279-1283.

[18] D. Oletic and V. Bilas, "Design of sensor node for air quality crowdsensing," 2015 IEEE Sensors Applications Symposium (SAS), Zadar, 2015, pp. 1-5.

[19] P. Dhar and P. Gupta, "Intelligent parking Cloud services based on IoT using MQTT protocol," 2016 International Conference on Automatic Control and Dynamic Optimization Techniques (ICACDOT), pp. 30-34, Pune, 2016.

[20] R. Y. Rodriquez, M. R. Julcapoma and R. A. Jacinto, "Network monitoring environmental quality in agriculture and pisciculture with low power sensor nodes based on Zigbee and GPRS technology," 2016 IEEE XXIII International Congress on Electronics, Electrical Engineering and Computing (INTERCON), pp. 1-6, Piura, 2016.

[21] X. Zhang; J. Du; C. Fan; D. Liu; J. Fang; L. Wang, "A wireless sensor monitoring node based on automatic tracking solar-powered panel for paddy field environment," IEEE Internet of Things Journal, vol.PP, no.99, pp.1$1,2017$.

[22] D. H. Kang et al., "Room Temperature Control and Fire Alarm/Suppression IoT Service Using MQTT on AWS," 2017 International Conference on Platform Technology and Service (PlatCon), pp. 1-5, Busan, 2017.

[23] A. Gomez; M. Magno; M. F. Lagadec; L. Benini, "Precise, Energy-Efficient Data Acquisition Architecture for Monitoring Radioactivity using Self-Sustainable Wireless Sensor Nodes," in IEEE Sensors Journal, vol.PP, no. 99, pp.1-1, 2017.

[24] J. E. Luzuriaga, J. C. Cano, C. Calafate, P. Manzoni, M. Perez and P. Boronat, "Handling mobility in IoT applications using the MQTT protocol," 2015 Internet Technologies and Applications (ITA), Wrexham, pp. 245-250, 2015.

[25] K. Grgic, I. Speh and I. Hedi, "A web-based IoT solution for monitoring data using MQTT protocol," 2016 International Conference on Smart Systems and Technologies (SST), Osijek, 2016, pp. 249-253.

[26] S. Ahmed, A. Topalov and N. Shakev, "A robotized wireless sensor network based on MQTT cloud computing," 2017 IEEE International Workshop of Electronics, Control, Measurement, Signals and their Application to Mechatronics (ECMSM), pp. 1-6, Donostia-San Sebastian, 2017.

[27] S. M. Kim, H. S. Choi and W. S. Rhee, "IoT home gateway for auto-configuration and management of MQTT devices," 2015 IEEE Conference on Wireless Sensors (ICWiSe), pp. 12-17, Melaka, 2015.

[28] A. Antonic, M. Marjanovic, P. Skoeir and I. P. Zarko, "Comparison of the CUPUS middleware and MQTT protocol for smart city services," 2015 13th International Conference on Telecommunications (ConTEL), pp. 1-8, Graz, 2015.

[29] T. Yokotani and Y. Sasaki, "Comparison with HTTP and MQTT on required network resources for IoT," 2016 International Conference on Control, Electronics, Renewable Energy and Communications (ICCEREC), pp. 1-6, Bandung, 2016.

[30] K. Rajaram and G. Susanth, "Emulation of IoT gateway for connecting sensor nodes in heterogenous networks," 2017 International Conference on Computer, Communication and Signal Processing (ICCCSP), pp.1-5, 2017.

[31] https://www.cloudmqtt.com.

[32] https://reliefweb.int/disaster/dr-2015-000180-vnm.

[33] Datasheet "Product specification", Product name S76S, Version F, Doc No 901-10201, AcSiP Technology Corp., Oct. 2016

[34] Hang Vu-Thanh, Thanh Ngo-Duc, Tan Phan-Vam, "Evolution of meteorological drought characteristics in Vietnam during the 1961-2007 period", Theoretical and Applied Climatological, Volume 118, Issue 3, p-p 368-370, November 2014.

[35] SK Acharya, G C Mishra, Karma P Kaleon, Lalu Das, "People's Perception on Climate Change and a Typical Hill Ecosystem of India”, Krishi Sanskriti Publications, p-p 10-11, New Delhi, 2015.

[36] Marius Lungu, Liliana Panaitescu and Simona Niţă, "Aridity, Climatic Risk Phenomenon in Dobrudja", Present environment and sustainable development, Vol. 5, no. 1, p-p 181-182, 2011. 


\section{BIBLIOGRAPHY OF AUTHORS}

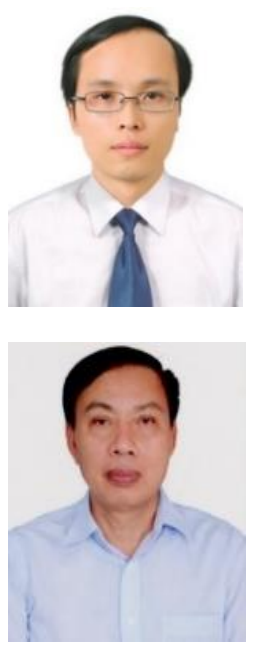

Van-Phuc Hoang was born in Hung Yen Province, Vietnam. He received $\mathrm{PhD}$ degree in Electronic Engineering from the University of Electro-Communications, Tokyo, Japan in 2012. $\mathrm{He}$ has worked as Postdoc researcher, visiting scholar at the University of ElectroCommunications, Tokyo, Japan and Universiy of Strathclyde, Glasgow, UK during the period of 2012-2014. He is working as an Associate Professor, Deputy Head of The Department of Microprocessor Engineering, Le Quy Don Technical University, Hanoi, Vietnam. His research interests include embedded systems for Internet of Things, VLSI architecture for digital signal processing, digital circuits and systems, low power IC design and hardware security.

Minh-Hong Nguyen was born in 1960. He received $\mathrm{PhD}$ degree in Automation Engineering from Le Quy Don Technical University, Hanoi, Vietnam. He is working as the Deputy Director of Center for Automation Engineering, Le Quy Don Technical University. His research interests include automation engineering, embedded systems for Internet of Things, VLSI architecture for digital signal processing, digital circuits and systems.

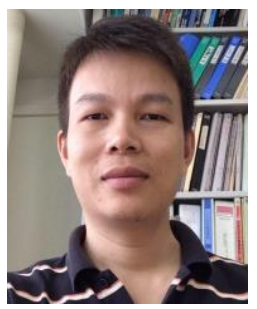

Thanh Quan Do was born in Hung Yen Province, Vietnam. He received PhD degree in Electronic Engineering Yokohama National University, Japan in 2017. His research interests include wireless communications, Internet of Things, digital circuits and systems, advanced digital signal processing algorithms.

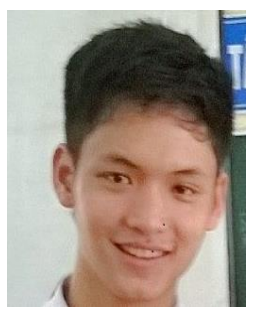

Dinh-Nhan Le is a Bachelor student of Le Quy Don Technical University, Hanoi, Vietnam. $\mathrm{He}$ was the leader of a student scientific research team receiving the Certificate of Merit from the university president for the excellent research achievement. His research interests include embedded systems for Internet of Things, automation engineering and advanced digital signal processing algorithms.

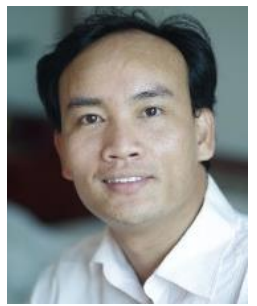

Du Duong Bui received PhD degree at Tokyo Metropolitan University, Japan in 2011. He has 14year experience in teaching, research and management in the areas of Water resources \& Climate change: Impacts and Adaptation; Disaster management including drought management, mitigating water-related risks; Water management policy and water governance. He is founder of the Vietnam Water Cooperation Initiative - a global platform hosted by Vietnam to promote water collaborations with worldwide partners. He has visit more than 20 countries around the world and published extensively, including peer-reviewed articles and book chapters. 